Instituto Internacional de Investigación y Desarrollo Tecnológico Educativo INDTEC, C.A.

DOI: https://doi.org/10.29394/scientific.issn.2542-2987.2017.2.3.7.138-154

OAI-PMH: http://www.indteca.com/ojs/index.php/Revista Scientific/oai

\title{
Jardín de Plantas Medicinales como Alternativa para Desarrollo de una Cultura Ambiental
}

Autora: Carmen Yusleiby Peña Rondón Universidad Pedagógica Experimental Libertador, UPEL yusleibycarla@gmail.com Barinas, Venezuela

\section{Resumen}

La presente investigación tiene como propósito implementar un jardín de plantas medicinales como alternativa para la formación de valores hacia una cultura ambiental, en la Escuela Técnica Agropecuaria Nacional "Francisco Tamayo" de la parroquia Barinitas municipio Bolívar estado Barinas. La misma se encuentra bajo el enfoque cualitativo, a través del método investigación-acción participante. Así mismo se apoya en la modalidad de campo y su diseño metodológico está estructurado en cuatro fases: (1) Reflexión inicial; (2) planificación; (3) puesta en práctica del plan y alcances y limitaciones; (4) reflexión en torno al proceso. Los informantes claves estarán representados por un docente, tres estudiantes, un representante, y un obrero quienes darán la información pertinente que servirán de base para la planificación y ejecución de las estrategias. Las técnicas de recolección de información serán la observación participante y la entrevista a profundidad. La técnica de análisis de la información será por la categorización para reducirla y así expresarla, describirla e interpretarla. Entre las consideraciones preliminares se espera los jóvenes adquirirán los valores para fortalecer el desarrollo hacia una cultura ambiental sustentable, permitirá brindar oportunidades de aprendizaje, donde vinculen la experiencia cognitiva con la vivencial, a través de prácticas de campo.

Palabras clave: cultura ambiental; valores; jardín; plantas medicinales. 


\title{
Medicinal Plant Gardens as an Option to the Development of an Environmental Culture
}

\begin{abstract}
This research aims to implement a medicinal plant gardens as an option to create values in an environmental culture in the Francisco Tamayo Technical School located in Barinitas parish, Bolívar municipality state Barinas. The approach of this study is qualitative thorough the participatory action research. Besides, it supports the field method and its methodological design is structured in four phases: (1) Reflection, (2) Planning, (3) Execution Plan, scopes and limitations (4) Reflection about all the process. The key people will be: (01) teacher, (03) students, (01) representative and a school worker who will give the accurate information to plan and execute the strategies. The technique of gathering information will be the participatory observation and the deep interview. The analysis techniques of gathering information will be the categorization to reduce, describe and interpretate it. Among the preliminary considerations are expected students get values to encourage the development of a sustainable environmental culture. It will also allow learning opportunities where involve the cognitive experience with the existential throughout field practices.
\end{abstract}

Keywords: environmental culture; values; garden; medicinal plants.

Date Received: 11-09-2016

Date Acceptance: $20-10-2016$ 


\section{Introducción}

Cuando el hombre apareció en el planeta estaba totalmente sometido a todas las fuerzas del ambiente natural, no solo en su entorno, sino también en sí mismo, porque desconocía las leyes y los mecanismos que rigen el funcionamiento de la naturaleza. Empezó entonces el largo camino desde el tiempo en que el hombre tenía que dedicar gran parte de sus energías para conseguir los alimentos necesarios, y para escapar de los peligros presentes, hasta el período actual en el cual, para sobrevivir el hombre tiene que dedicar una parte de sus energías a reducir y a corregir los riesgos con los cuales su misma presencia y actividad amenazan tanto al ambiente natural como al que él mismo ha creado. Al respecto, se señala:

La crisis ambiental se torna esencialmente cultural, para rebasar la crisis y volver a un punto de relación armónica entre la naturaleza y la sociedad se requieren nuevas concepciones donde se integran valores, concepciones del mundo, ideologías, tradiciones, conocimientos científicos y empíricos (Bermúdez, 2004, pág. 1)

Actualmente, en los países de Latinoamérica, ésta crisis ha trascendido en el desarrollo económico y cultural, debido a que los planes de desarrollo precisan la búsqueda de nuevos recursos vegetales, que contribuyan a satisfacer las necesidades básicas de la sociedad, así como obtener materias primas para el desarrollo económico de las industrias y las sociedades; así mismo programas que propicien la introducción de nuevos cultivares adaptados a las características ecológicas y tecnológicas de las distintas regiones agrícolas, sin causar graves impactos al medio.

Por consiguiente, para desarrollar propuestas educativas, han de tenerse en cuenta los fundamentos epistemológicos y sociopolíticos del modo de pensar, la relación sociedad-naturaleza por el hombre hoy. La educación responde a objetivos concretos, y ello se delimita por los entornos sociales específicos, donde los elementos cognitivos y sociopolíticos globales toman 
cuerpo con las tradiciones locales (comunitarias y nacionales) y se plasman en actos educativos posibles.

Por otra parte, el presente trabajo está estructurado de la siguiente manera:

Momento I: Plantea la contextualización del problema objeto de estudio, se desarrolla el propósito general, específicos y la justificación.

Momento II: Está referido a las perspectivas teóricas, donde se presentan los antecedentes, teorías, enfoques que sustentan la investigación

Momento III: Se plantea el marco metodológico, donde se especifica el tipo de investigación, la metodología, técnicas de recolección de la información a recabar.

\subsection{Propósitos de la Investigación}

\subsubsection{Propósito General:}

Implementar un jardín de plantas medicinales como alternativa para la formación de valores hacia una cultura ambiental, en la Escuela Técnica Agropecuaria Nacional "Francisco Tamayo" de la parroquia Barinitas, municipio Bolívar, estado Barinas.

\subsubsection{Propósitos Específicos:}

- Diagnosticar los valores ambientales que se vive en la Escuela Técnica Agropecuaria Nacional "Francisco Tamayo" de la parroquia Barinitas, municipio Bolívar, estado Barinas.

- Desarrollar un plan de acción para la implementación de un jardín de plantas medicinales, su utilización y la formación de valores hacia una cultura ambiental en la Escuela Técnica Agropecuaria Nacional "Francisco Tamayo" de la parroquia Barinitas, municipio Bolívar, estado Barinas.

- Ejecutar un plan de acción para la implementación de un jardín de 
plantas medicinales como estrategia formativa de valores hacia una cultura ambiental.

- Evaluar los resultados y el impacto socio-cultural en la comunidad que surge de la implementación del Jardín de Plantas Medicinales con la participación de todos los actores, estudiantes, profesores y familia en la institución.

\section{Fundamentos Teóricos}

Estas comprenden un conjunto de conceptos y proposiciones que constituyen un punto de vista determinado dirigidos a explicar el fenómeno planteado.

\subsection{Hierbas Medicinales}

Las hierbas medicinales, según ConceptoDefinición (2004), la definen como:

Las hierbas medicinales son distintas plantas o vegetales de diferentes especies, las cuales de acuerdo a su estructura química y composición estructurales pueden ejercer soluciones curativas para distintas lesiones del organismo que posiblemente presenten los seres humanos, patologías como problemas de digestión, lesiones cutáneas, problemas respiratorios, así como también pueden ser utilizados para problemas de insomnio e intranquilidad. (pág. 1)

Al respecto, el cultivo de plantas medicinales le brindará al estudiante el beneficio de poder aliviar alguna dolencia, al tener el conocimiento de sus propiedades, así como el valor ambiental ya que practicará las labores culturales desde que se siembra utilizando técnicas agroecológicas.

Por otra, Olaya (2003), las plantas medicinales son vegetales, que elaboran los principios activos, que tienen actividad benéfica o perjudicial sobre el organismo, sirven para disminuir o neutralizar enfermedades, alivian y devuelven la salud perdida. 
En este sentido las plantas medicinales se utilizan como medicamentos para el tratamiento de alguna afección o enfermedad que padece un individuo o animal. Muchas de ellas se cultivan en jardines botánico, al respecto, Vovides, Linares y Bye (2010), la define como:

Son jardines o parques recreativos, temáticos, educativos 0 , sencillamente, muy bonitos y diversos que pueden tener algunas colecciones de flora, pero no son botánicos porque no cumplen con la tarea de mantener colecciones vivas de plantas debidamente documentadas e inventariadas para propósitos de investigación científica, educación y conservación (pág. 17)

Cabe destacar, que el jardín de plantas medicinales es un excelente recurso en donde los estudiantes desarrollen múltiples experiencias acerca de su entorno natural, poner en práctica actitudes, hábitos de cuidado y responsabilidad ambiental; llevando a cabo los ejes transversales de valores y ambiente relacionando temas como el consumo, la basura, el reciclaje, la salud y el desarrollo de los pueblos.

Nuestros antepasados poseían grandes conocimientos sobre las plantas medicinales que utilizaban para curar sus enfermedades, y que lamentablemente, a través del tiempo, han ido desapareciendo, razones es el auge de los fármacos, por lo que la población ha venido perdiendo la costumbre de cultivar este tipo de plantas.

Por esta razón el jardín de plantas medicinales es un incentivo para que los estudiantes sean los protagonistas del ambiente natural, realizando diferentes actividades que los motiven a desarrollar sus habilidades, destrezas y se promueva los valores ambientales.

\subsection{Diseño de un jardín}

El diseño de los jardines consiste desde el bosquejo de las plantas hasta su cultivo mismo. En concordancia, Ballesta (2006) expone, antes de diseñar el jardín debemos tener algunos conocimientos elementales sobre la 
naturaleza del suelo, de la exposición solar adecuada a la especie para evitar quemaduras.

Ballesta, considera que existen diversos pasos para establecer un jardín de plantas medicinales, entre ellos están:

1. Construcción: se trata de un proceso laborioso en los que los pasos a seguir son los siguientes:

1.1. Replanteo de plano sobre el terreno.

1.2. Movimientos de tierra.

1.3. Labores preparatorias de profundidad, el suelo debe tener $30 \mathrm{~cm}$ como mínimo de fondo.

1.4. Nivelar la superficie.

1.5. Diferenciar el espacio dedicado al cultivo.

1.6. Instalar el riego.

1.7. Trazar accesos y caminos.

1.8. Realizar las labores preparatorias superficiales y mejorar sus condiciones nutritivas.

2. El Agua: Es conveniente, el aprovechamiento del agua sea el máximo aportando a la planta la dosis ajustada a sus necesidades, y solamente donde sea necesaria. Las necesidades de una planta dependen fundamentalmente de la especie a la que pertenece, pero también de las condiciones del cultivo.

3. Labrar el Terreno: Para labrar el jardín en sí, antes de aplicar cualquier material orgánico, simplemente con una pala, sacando y removiendo la tierra para que se deshaga, con el arado de la tierra buscamos mejorar las condiciones del suelo. 


\subsection{Cómo Cultivar el jardín}

Según el mismo autor señala, al adquirir una planta debemos tener presente que se trata de un ser vivo y, por lo tanto, sensible a los cambios en su condición de vida y el medio que la rodea. De allí entonces que se deben considerar ciertas precauciones al momento de la adquisición de plantas y plantación, tales como:

- Cada ejemplar ha de estar etiquetado individualmente, con el nombre completo y correcto, especificando la variedad.

- Las hojas han de ser numerosas y de aspecto saludable, no debe haber hojas caídas alrededor de la planta.

- No deben presentar heridas ni síntomas de plagas o enfermedades.

- El cepellón debe estar integro, sujeto a las raíces, húmedo y dejar el cuello al aire.

- Las raíces no deben asomar por fuera del contenedor y no deben presentar espiralización.

- Los hoyos deben prepararse unos días antes de la plantación para que el terreno se airee, como regla general se puede considerar que el hoyo debe ser un tercio mayor que el contenedor en que se encuentra la planta, cuanto mayor sea el agujero mejor y más rápido será el desarrollo del sistema radicular, y por tanto, de la planta.

- Tras terminar de llenar el hoyo con tierra y compactarla, se realiza un riego abundante para humedecer toda la tierra aportada.

Dentro del jardín de plantas medicinales se pueden establecer una gran diversidad de cultivos como:

- Yerbas aromáticas y comestibles.

- Ornamentales.

- Frutales. 
Cabe destacar, que las plantas medicinales son uno de los remedios totalmente caseros y naturales que más beneficios y propiedades aportan al organismo, por ello, resulta útil saber cuáles son los beneficios, así como sus propiedades. Entre ellas se destacan:

- Toronjil: sedante, antidepresivo, relajante, digestivo, antiviral, carminativo.

- Romero: astringente, digestivo, antiséptico, carminativo, diurético, antidepresivo, estimulante circulatorio.

- Cayena: esta planta se utiliza como infusión sedante.

- El orégano es rico en hierro, magnesio, calcio y potasio.

- Zabila: Cicatrizar, inflamaciones de la piel, mejorar estreñimiento, mejorar función vesícula biliar y antirreumática.

- Limón: inflamaciones, astringente, gases, disentería, colirio, gota, reumatismo, cálculos, hipertensión, gripes, resfriados, jaquecas, diarreas, reductor de grasa

- Girasol: reumatismo, afrodisíaco, febrífugas, gripes, paludismo, cálculos, gastritis, cefaleas vasculares, diuréticas, desinfectantes, bronquitis, tosferina.

\subsection{Educación Ambiental}

Labrador, y Del Valle (1995), quienes resumieron los alcances del Seminario organizada por las Naciones Unidas celebrado en Tbilisi en septiembre de 1977, señala que la educación ambiental constituye el proceso de internalizar los conceptos y valores necesarios para planificar actividades con la finalidad de que el ser humano comprenda la relación que existe entre él, su cultura y el ambiente.

Por otro lado, Alberto (2001), expresa:

La educación ambiental debe contemplar al individuo desde una perspectiva ecológica, como ser integrante de un 
ecosistema, tiene que ser concebida como una educación permanente, es decir, como un proceso que se inicia en los primeros estadíos escolares y que no debe concluir jamás (pág. 68)

Por ello, surge así la necesidad de tener un mejor conocimiento del ambiente, de adquirir habilidades e iniciar acciones tendientes a un uso sensato sin perturbar el equilibrio ecológico.

Asimismo, la educación ambiental pretende fundamentalmente concienciar y crear conocimientos que permitan enfrentar los problemas ambientales, así como, mejorar actitudes que accedan una verdadera participación de los individuos en la protección y mejoramiento del mismo.

En tal sentido, se debe promover la participación activa de los estudiantes favoreciendo una repuesta global, mediante la adquisición de conocimientos conceptuales y el desarrollo de actitudes, capacidades y destrezas. Todo esto, requiere de un docente creador que adapte el currículo, organizando situaciones ricas de aprendizaje, ligadas a la experiencia del estudiante.

\subsection{Valores Ambientales.}

Según Artieda (1999): los valores ambientales se enfocan en ayudar a la gente a madurar hacia una moralidad que fusione el amor y la justicia, que se preocupe por los demás y por el medio ambiente.

Asimismo, los valores ambientales se inculcan por diferentes medios (y no sólo dentro de los procesos educativos formales), produciendo efectos educativos. Estos valores van desde los principios ecológicos generales (comportamiento en armonía con la naturaleza) y una nueva ética política (apertura hacia una pluralidad política y la tolerancia hacia el otro) hasta los nuevos derechos colectivos y los intereses sociales asociados a la reapropiación de la naturaleza y la redefinición de estilos de vida diversos, que 
rompen con la homogeneidad y centralización del poder en el orden económico, político, cultural y dominante.

De este modo, con la implementación del jardín de plantas medicinales se espera inculcar los valores ambientales que no sólo produzca una armonía con la naturaleza, sino que despierte en los estudiantes el interés por ejecutar proyectos de orden económico, cultural y político.

Bermúdez (2004), hace referencia a la definición de la educación ambiental realizada por la Unión Internacional para la Conservación de la Naturaleza (IUCN) en el año 1970 como aquel proceso que consiste en reconocer valores y clasificar concepto con el objeto de desarrollar las actitudes necesarias para comprender y apreciar las interrelaciones entre el hombre, su cultura y su medio físico.

\section{Metodología}

\subsection{Enfoque de la Investigación}

El estudio se encuentra enfocado bajo el paradigma de investigación cualitativa. Al respecto, Rojas (2010) señala que: "la investigación cualitativa se orienta hacia la construcción de un conocimiento acerca de la realidad social y cultural a partir de la descripción e interpretación de las perspectivas de los sujetos involucrados" (pág. 57).

Por lo antes expuesto, la comunidad estudiantil a través de la creación de un jardín de plantas medicinales fomentará valores dirigidos al rescate de la cultura ambiental, reconquista de las prácticas ancestrales realizadas en los hogares en armonía con su entorno.

\subsection{Tipo de Investigación}

En relación con el diseño del estudio se empleará la metodología de la investigación Acción Participativa que según Kemmis y McTaggart (1998) se caracteriza como la búsqueda conjunta promovida por sujetos en ciertas 
situaciones sociales con la finalidad de mejorar la racionalidad de sus prácticas sociales o educativas.

El estudio a través de la investigación acción proporcionará no sólo el conocimiento del cultivo de plantas medicinales, sino también la adquisición de valores de compromiso, justicia y respeto hacia el ambiente. De igual manera, el uso y beneficio de las plantas para la salud y el rescate de la cultura ambientalista.

Asimismo, existen diversos enfoques de investigación-acción, en el presente estudio se aplica el método de investigación participativa, la cual posee las siguientes características:

(a) Forma parte de una situación específica en la cual se ha detectado una problemática. (b) Desarrolla un proceso dialéctico en el cual la acción genera conocimiento y la investigación conduce a la transformación. (c) En el proceso trabajan de forma conjunta y mancomunada los integrantes de la comunidad en estudio, los investigadores, promotores y animadores. (d) La dinámica de participación genera un compromiso, tanto del investigador como del resto de los participantes (Rojas, 2010, (pág. 51)

De igual manera, existen cuatro momentos en la investigación-acción, los cuales son:

(1) Reflexión inicial acerca de la situación a la luz de la preocupación temática; (2) planificación conjunta de actividades y estrategias para mejorar la situación; (3) puesta en práctica del plan y alcances y limitaciones; (4) reflexión en torno al proceso los resultados con miras a reconsiderar la preocupación temática, las oportunidades y restricciones, los logros e implicaciones futuras e introducir los cambios que se consideren pertinentes para volver a comenzar el ciclo. (Rojas, 2010, pág. 52)

\subsection{Técnica de Recolección de Información}

De acuerdo con Albert (2007) "en el enfoque cualitativo, la recolección de datos ocurre completamente en los ambientes naturales y cotidianos de los 
sujetos e implica dos fases o etapas: (a) inmersión inicial en el campo y (b) recolección de los datos para el análisis" (pág. 231). Para la presente investigación, se utilizará la observación participante en donde el autor mencionado señala: "Se trata de una técnica de recolección de datos que tiene como propósito explorar y describir ambientes...implica adentrarse en profundidad, en situaciones sociales y mantener un rol activo, pendiente de los detalles, situaciones, sucesos, eventos e interacciones". (pág. 232)

Igualmente, se utilizará la entrevista que Rojas (2010) la define como: "un encuentro en el cual el entrevistador intenta obtener información, opiniones o creencias de una o varias personas" (pág. 85). Para esta investigación la entrevista a utilizar es la de profundidad, en la cual según Albert (2007): el entrevistador sugiere algunos temas importantes con la finalidad de que los sujetos de investigación expresen libremente sus ideologías y sentimientos sobre dichos contenidos, ésta es conversacional y sin juicio de valor.

\subsection{Técnicas de Análisis de la Información}

\subsubsection{La Categorización}

Con la finalidad de expresar y describir la información para que sea perceptible y significativa para otras personas, se busca reducirla, respondiendo a una estructura sistemática. Con relación a lo anterior, Cisterna (2005) expone que uno de los elementos básicos para analizar la información es la elaboración de temas a partir de los cuales se recoge y organiza. Asimismo, en la investigación cualitativa los informantes claves conforman uno de los recursos indispensables, para lo cual Martínez (1991) son "personas con conocimientos especiales, status y buena capacidad de información" (pág. 56). En esta investigación los informantes claves están representados por un docente, tres estudiantes, un representante, y un obrero quienes darán la información pertinente que servirán de base para la ejecución del plan acción. 


\subsubsection{Credibilidad y Fiabilidad}

La credibilidad se logra cuando el investigador, a través de observaciones y entrevistas con los participantes del estudio, recolecta información que produce hallazgos que son reconocidos por los informantes como una verdadera aproximación sobre lo que ellos piensan y sienten.

La credibilidad se puede alcanzar debido a que los investigadores, para confirmar los hallazgos y revisar algunos datos particulares, vuelven a los informantes durante la recolección de la información. Según Castillo y Vásquez (2003): a los sujetos de investigación les gusta participar en la revisión para reafirmar su participación y que los hallazgos sean lo más creíbles y precisos.

La Credibilidad o validez interna está basada en varias técnicas, para el presente estudio se aplica la triangulación la cual se define según Guba y Lincoln (1982) como una asimilación traída de la ingeniería referida a los ángulos que dan fuerza a una estructura.

Rojas (2010) dice que, así como no hay validez sin confiabilidad, tampoco hay credibilidad sin confiabilidad.

\subsubsection{Triangulación}

Dentro de esta conceptuación existe la triangulación de fuentes, la cual permite confirmar la información suministrada por los distintos sujetos.

Según Guba y Lincoln (1982), es una temática que se utiliza al establecer la independencia entre las observaciones e interpretaciones recabadas sin haber participado en su recolección y luego confrontar los resultados. Ésta se puede establecer a través del control doble ciego, que consiste en que dos investigadores examinan por cuenta propia las informaciones recabadas para su confrontación sin haber participado en su recolección. 


\section{Resultados y Conclusiones}

Los resultados y conclusiones no se presentan ya que se encuentran en análisis de estudio. Sin embargo, se presenta una serie de estrategias que se han derivado del análisis que hasta ahora se ha realizado y que servirán de base para la ejecución del plan de acción. Entre ellas se destacan:

- Talleres, foros y video conferencias que orienten a los padres, estudiantes, directivos y comunidad en general sobre el uso de las plantas medicinales.

- Jornadas de concienciación sobre la importancia del uso de las plantas medicinales.

- Construcción de un vivero donde se cultiven plantas medicinales con técnicas agroecológicas para fomentar los valores ambientalistas.

- Elaboración de catálogos donde se identifiquen las plantas con su nombre científico, propiedades, características, origen y beneficios.

\section{Referencias}

Albert, M. (2007). La Investigación Educativa. Claves Teóricas. España: McGraw-Hill.

Alberto, O. (2001). Medio ambiente y educación: Capacitación en educación ambiental para docentes. 2da edición, ISBN: 987-538026-1. Argentina: Ediciones Novedades Educativas.

Artieda, G. (1999). Educación ambiental: Cuestiones básicas. Lurralde, 22, 279-298, e-ISSN: 1697-3070. España.

Ballesta, C. (2006). Manual de Jardinería. Ediciones Ceac. S.A. Bermúdez, A. (2004). Caracterización del comercio de plantas medicinales en los mercados populares de Caracas, Venezuela. 2do. Seminario Iberoamericano de Comercialización de Plantas Medicinales y Aromáticas. Bogotá, Colombia. 59-70.

Castillo, E., y Vásquez, M. (2003). El rigor metodológico en la investigación 
cualitativa. Colombia Médica, 34(3), 164-167, e-ISSN: 0120-8322.

Recuperado de: https://www.redalyc.org/articulo.oa?id=28334309

Cisterna, F. (2005). Categorización y Triangulación. C.A-ORIAL ediciones.

ConceptoDefinición (2004). Hierbas medicinales. [Diccionario en línea].

Recuperado de: http://conceptodefinicion.de/hierbas-medicinales/

Guba, G. E. y Lincoln, Y. S. (1982). Effective evaluation. San Francisco: Jossey Bass Publishers.

Kemmis, S. y McTaggart, R. (1988). Cómo Planificar la investigación Acción. Barcelona: ALERTES.

Labrador, C., y Del Valle, Á (1995). La Educación Medioambiental en los documentos internacionales: Notas para un estudio comparado. Revista Complutense de Educación, 6(2). Madrid: Servicio de Publicaciones de la Universidad Complutense.

Martínez, M. (1991). La investigación cualitativa etnográfica en educación: Manual teórico - práctico. Venezuela: Texto.

Olaya, J. (2003). Guía de plantas y productos medicinales. Editorial Luis Enrique Acero Duarte.

Rojas, B. (2010). Investigación Cualitativa. Segunda edición Caracas. FEDUPEL.

Vovides, A., Linares, E., y Bye, R. (2010). Jardines botánicos de México: historia y perspectivas. ISBN: 978-607-7579-18-2. México: Secretaría de Educación de Veracruz. 


\section{Carmen Yusleiby Peña Rondón}

e-mail: yusleibycarla@gmail.com

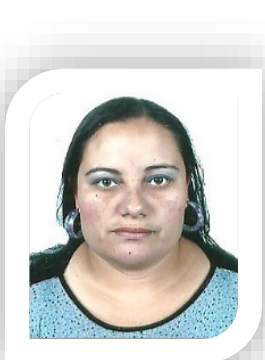

Nacida en el Municipio Bolívar, Parroquia Barinitas Del Estado Barinas, Venezuela. Cursa estudios de magister en educación ambiente y desarrollo en la Universidad Pedagógica Experimental Libertador Instituto Del Mejoramiento Profesional Del Magisterio núcleo académico Barinas. Licenciada en educación mención física de la universidad experimental de los llanos Ezequiel Zamora (2010). Actualmente se desempeña como docente de aula en la institución educativa Escuela Técnica Agropecuaria Nacional Francisco Tamayo desde el 2013 hasta la fecha.

Con participación en diferentes talleres dirigidos hacia el área de educación que fortalecen la formación docente.

El contenido de este manuscrito se difunde bajo una Licencia de Creative Commons ReconocimientoNoComercial-Compartirlgual 4.0 Internacional 